\title{
Presentación y manejo de las cardiopatías congénitas en el primer año de edad
}

\author{
Presentation and management of congenital heart disease in the first year of age
}

Rocio A. Peña-Juárez ${ }^{1 *}$, Carlos Corona-Villalobos², Miguel Medina-Andrade', Luis Garrido-García², Carlos Gutierrez-Torpey y Moisés Mier-Martínez ${ }^{2}$

${ }^{1}$ Servicio de Cardiología Pediátrica, Hospital General de Occidente, Zapopan, Jal.; ${ }^{2}$ Servicio de Cardiología, Instituto Nacional de Pediatría, Ciudad de México, México

\section{Resumen}

Las cardiopatías congénitas (CC) se definen como una anomalía estructural del corazón o de los grandes vasos intratorácicos. Constituyen la malformación congénita más frecuente al nacimiento. Al menos un tercio de los pacientes requieren algún tipo de intervención antes del año de edad. Las manifestaciones clínicas de las cardiopatías en la etapa neonatal se presentan con un amplio contexto clínico y se pueden confundir con problemas a nivel pulmonar o infeccioso, lo que dificulta su diagnóstico y con ello contribuyendo de forma importante a la mortalidad y morbilidad de estos pacientes, ya que se retrasa el diagnóstico y manejo oportuno. El monitoreo por oximetría de pulso en el periodo neonatal se utiliza actualmente como método diagnóstico para la detección de cardiopatías congénitas críticas; a pesar de que las detecta en forma temprana, en muchos países aún no se lleva a cabo. El objetivo de este artículo es ofrecer un panorama general de la presentación clínica, aspectos diagnósticos y manejo inicial de las CC en el primer año de edad que pueda ser de utilidad a los médicos de primer contacto para mejorar la atención en este grupo de pacientes.

Palabras clave: Cardiopatía congénita. Técnica diagnóstica cardiovascular. Prostaglandinas.

\begin{abstract}
Congenital heart disease (CHD) is defined as a structural abnormality of the heart or large intrathoracic vessels. They constitute the most frequent congenital malformation at birth. At least one third of patients require some type of intervention before the year of age. The clinical manifestations of heart disease in the neonatal stage are presented with a wide clinical context and can be confused with problems at the pulmonary or infectious level making difficult to diagnose them and thereby contributing significantly to the mortality and morbility of these patients since the diagnosis is delayed and timely handling. Pulse oximetry monitoring in the neonatal period is currently used as a diagnostic method for the detection of critical congenital heart disease. Although it detects them early, in many countries it is not yet carried out. The objective of this article is to offer an overview of the clinical presentation, diagnostic aspect and initial management of $\mathrm{CHD}$ in the first year of age that may be useful to first contact physicians to improve the management of this group of patients.
\end{abstract}

Key words: Congenital heart. Cardiovascular diagnostic technique. Prostaglandins.

\footnotetext{
Correspondencia:

*Roció A. Peña-Juárez

E-mail: alepejz@gmail.com

Disponible en internet: 23-11-2020 Arch Cardiol Mex. 2021;91(3):337-346 www.archivoscardiologia.com 1405-9940 / @ 2020 Instituto Nacional de Cardiología Ignacio Chávez. Publicado por Permanyer. Este es un artículo open access bajo la licencia CC BY-NC-ND (http://creativecommons.org/licenses/by-nc-nd/4.0/).

Fecha de recepción: 02-03-2020 Fecha de aceptación: 21-08-2020 DOI: $10.24875 /$ ACM.20000113
} 


\section{Introducción}

La definición de cardiopatías congénitas (CC) varía ampliamente entre los informes epidemiológicos de la enfermedad. En esta revisión nos adherimos a la definición propuesta por Mitchell, et al., los cuales la refieren como «una anomalía estructural grave del corazón o los grandes vasos intratorácicos que es real o posiblemente de importancia funcional» ${ }^{1}$. Constituyen la malformación congénita más frecuente ${ }^{2}$, con una incidencia de 2.5 a 3 por cada 1,000 nacimientos ${ }^{3,4}$. En el 2000 la prevalencia global era de 11.9 por cada 1,000 nacimientos $^{5}$. Al menos un tercio requerirán algún tipo de intervención en el primer mes de vida ${ }^{6}$ con un alto riesgo de muerte sin un diagnóstico oportuno. Su presentación clínica tiene un amplio contexto, la presentación cardinal de cianosis, shock e insuficiencia cardiaca pueden confundirse con problemas a nivel pulmonar o infeccioso, lo que retrasa su diagnóstico y, por lo tanto, los médicos que atienden a estos pacientes deben establecer una amplia gama de diagnósticos diferenciales y considerar las CC cuando se maneja un neonato críticamente enfermo ${ }^{7}$. El monitoreo por oximetría de pulso se utiliza como método de tamizaje para la detección de cardiopatías congénitas críticas $(\mathrm{CCC})^{8}$, sin embargo, a pesar de que la Academia Americana de Pediatría (AAP) y la Asociación Americana del Corazón (AHA) lo recomiendan ${ }^{9}$ en muchos hospitales no se cuenta con el material que se requiere para realizarlo. Los avances en el tratamiento de los niños con CC han sido notables, con una disminución importante de la mortalidad quirúrgica (del 15\% en 1990 al 5\% en el año 2000), secundaria a la detección temprana, técnicas quirúrgicas y atención preoperatoria y postoperatoria ${ }^{10}$. Sin embargo, en muchos hospitales no se cuenta con el equipo multidisciplinario para su atención. Es por ello que los médicos que son el primer contacto de atención de estos pacientes requieren capacitación para la detección y manejo oportuno de las CC. El objetivo de este artículo es ofrecer un panorama general de la clínica, diagnóstico y manejo inicial de las CC que pueda ser de utilidad para mejorar la atención de estos pacientes. Para fines prácticos, el artículo se dividirá en dos partes: la primera incluye la etapa neonatal y la segunda, la etapa lactante.

\section{Etapa neonatal}

\section{¿Qué hacer con la sospecha de un recién nacido con cardiopatía congénita?}

Es primordial obtener una anamnesis adecuada, conocer las características del embarazo y parto. Realizar una exploración física completa, incluyendo presión arterial y palpación de pulsos en las cuatro extremidades. Si cuenta con algún dato de CC, existen pruebas de tamizaje; los dos métodos más utilizados son:

- Oximetría de pulso. Se realiza en recién nacidos asintomáticos entre las 24 a 48 horas de vida o una hora previa a su egreso. Su objetivo es detectar $\mathrm{CCC}$, es una herramienta no invasiva y de bajo costo ${ }^{9}$. La prueba consiste en la medición de oximetría de la extremidad superior derecha y cualquier extremidad inferior (preductal y posductal); sin embargo, existen cardiopatías con arco aórtico derecho, por lo que se recomienda medir la saturación de oxígeno $\left(\mathrm{SaO}_{2}\right)$ en ambos brazos y cualquier pie. Un resultado es anormal si se presenta: a) cualquier saturación $<90 \%$; b) $\mathrm{SaO}_{2}<95 \%$ en ambas extremidades en tres medidas separadas por 1 hora, o c) diferencia absoluta $>3 \%$ entre la mano y el pie en las tres mediciones, cada una separada por una hora ${ }^{9,11}$.

- Prueba de hiperoxia. Se realiza en casos donde se requiere diferenciar entre patología pulmonar 0 cardiaca. Los niños con enfermedad pulmonar presentan disminución en la concentración de oxígeno alveolar y muchas veces se incrementa el gradiente alveolar-arteriolar, lo cual no se observa en las CC $^{12}$. Se puede realizar la prueba de hiperoxia para diferenciar la causa de la desaturación, la cual está indicada en neonatos con saturaciones < $95 \%$. El objetivo es estudiar la respuesta de la presión parcial de oxígeno $\left(\mathrm{pO}_{2}\right)$ arterial tras la administración de oxígeno al $100 \%$ por un periodo de 10 minutos. Debe obtenerse una medición basal de la $\mathrm{pO}_{2}$ de la arterial radial derecha (preductal) y de una arteria de la extremidad inferior (posductal) cuando el niño respira aire ambiente (fracción de oxígeno inspirado $\left[\mathrm{FiO}^{2}\right.$ $21 \%)$ y posteriormente una nueva medición tras la administración de oxígeno al 100\% ( $\left.\mathrm{FiO}_{2}, 100 \%\right)$. Cuando el origen de la cianosis es pulmonar, la $\mathrm{pO}_{2}$ aumenta hasta $100 \mathrm{mmHg}$. Pero con un cortocircuito intracardiaco de derecha a izquierda la $\mathrm{pO}_{2}$ arterial no supera los $100 \mathrm{mmHg}$ y el aumento no es superior a $10-30 \mathrm{mmHg}$. Una $\mathrm{pO}_{2}$ menor a $70 \mathrm{mmHg}$, elevaciones menores de $30 \mathrm{mmHg} \circ \mathrm{SaO}_{2}$ sin cambio orienta francamente hacia una causa cardiogénica; raramente las CCC presentan un incremento en la concentración de oxígeno arterial a más de $150 \mathrm{mmHg}$ cuando se exponen al $100 \%$ de oxígeno. Aunque la $\mathrm{FiO}_{2}$ al $100 \%$ solo se logra con el paciente intubado, se cuenta con un test práctico donde se administra oxígeno a 10 litros/minuto por 10 minutos para valorar la respuesta de la saturación. Si la $\mathrm{SaO}_{2}$ por 
oximetría no incrementa a más del 95\%, la medición por gasometría arterial no es necesaria ${ }^{12}$.

\section{¿Qué cardiopatía tiene?}

Existen múltiples clasificaciones de las CC, sin embargo, para entender los tipos de CC y su manejo se requiere tomar en cuenta algunos puntos fisiológicos cardiovasculares básicos. Los principios hemodinámicos de la fisiología cardiaca se basan en los principios básicos de la mecánica de fluidos e hidrodinámica. Estos conceptos son fundamentales para entender las relaciones entre circulación pulmonar y sistémica, que es crucial para la atención adecuada de las $\mathrm{CC}^{13}$.

El gasto cardiaco se define como el volumen que bombea el corazón por unidad de tiempo; dos de sus componentes básicos son el flujo de sangre sistémico y pulmonar, siendo el cociente entre ambos de 1 . El método de referencia para la medición del cociente flujo pulmonar y flujo sistémico (Qp:Qs) es el método de Fick, que consiste en la medida de la concentración de oxígeno de muestras de sangre obtenida mediante catéter de distintos puntos de la circulación pulmonar y sistémica tras 10 minutos de respiración con oxígeno al $21 \%$, calculándose:

$\mathrm{Q}(\mathrm{l} / \mathrm{min})=$ Consumo de $\mathrm{O}_{2}(\mathrm{ml} / \mathrm{min}) /$ Diferencia arteriovenosa de $\mathrm{O}_{2}(\mathrm{ml} / \mathrm{l})$

De esta manera se puede caracterizar la disminución o incremento en la concentración de oxígeno secundario a un cortocircuito intrapulmonar, intracardiaco o por alteración de ventilación/perfusión. La medida del cociente entre el gasto pulmonar y el sistémico nos informa sobre la integridad de la independencia entre estas dos circulaciones ${ }^{14}$.

- Qs $\rightarrow$ flujo sistémico.

- Qp $\rightarrow$ flujo pulmonar.

- Qp:Qs $\rightarrow$ relación de flujos:

- Qp:Qs = $1 \rightarrow$ normal.

- Qp:Qs < $1 \rightarrow$ cardiopatías con flujo pulmonar disminuido (alteración en la continuidad del flujo ventriculopulmonar y permeabilidad del conducto arterioso) $^{14,15}$.

- Qp:Qs > $1 \rightarrow$ CC con flujo pulmonar aumentado, existe paso de sangre sistémica a la circulación pulmonar.

Tradicionalmente se nos enseñan las $\mathrm{T}$ y $\mathrm{H}$ de las $\mathrm{CC}$ cianóticas por sus siglas en inglés. Incluyen: atresia tricuspídea (TA), tetralogía de Fallot (TOF), transposición de las grandes arterias (TGA), tronco arterioso (TAC), conexión anómala total de venas pulmonares (TAPVR) y síndrome de ventrículo izquierdo hipoplásico
$(\mathrm{HLHS})^{16}$. Sin embargo, la variedad de las lesiones que presentan cianosis excede los límites de esta lista, por lo que es mejor que se enfatice en la comprensión de la fisiopatología de las CC mediante el uso de la presentación clínica para reducir los diagnósticos diferenciales y mejorar el manejo. La clasificación con base en la clínica y manejo se enlistan en la figura 1. Para ello se divide a los pacientes en dos grandes grupos, cada uno con subtipos:

El primer paso es determinar la presencia o no de cianosis:

- Cianosis. Es la coloración azulada de piel y mucosas secundaria a oxigenación deficiente por causa cardiaca o pulmonar. Es causada por la presencia de sangre desoxigenada a nivel capilar. Los neonatos pueden presentar cianosis periférica transitoria (acrocianosis), que puede ocurrir en niños con anatomía y función cardiaca normal como resultado de condiciones benignas como exposición al frío, y otras condiciones graves no cardiacas, como sepsis. La cianosis central incluye membranas mucosas, labios o tronco; siempre es patológica ${ }^{17}$. La cianosis depende del grado de desaturación arterial en primer lugar, y de la cantidad total de hemoglobina y del volumen minuto cardiaco. La interrelación de estos tres factores puede ser asimilada con la siguiente fórmula matemática:

$$
\frac{- \text { Hemoglobina }=\mathrm{K}}{-\mathrm{VMC} \times \mathrm{SaO} 2}
$$

Hemoglobina $=\mathrm{K}$

Volumen minuto cardiaco $\times \mathrm{SaO}_{2}$

Donde la cianosis estará directamente relacionada con la cantidad total de hemoglobina e inversamente con el volumen minuto cardiaco y con la saturación arterial de oxígeno. Esto explica la hipótesis de Lundsgaard y van Slyke, que consideran que debe haber 5 gramos de hemoglobina reducida en el capilar periférico para que sea observada ${ }^{1}$. En términos prácticos los desplazamientos de la curva de la disociación de la hemoglobina hacen inexacto el diagnóstico de la cianosis a partir de la coloración de piel, por ello es sumamente importante realizar la prueba de oximetría para evitar subdiagnosticar CCC.

El recién nacido con cardiopatía cianótica requiere manejo inmediato incluso mientras se espera la valoración cardiológica o su traslado. Los recién nacidos con CC cianótica se dividen en dos categorías:

- Cianosis con choque. Presentan importante compromiso cardiovascular y constituye una emergencia neonatal. Son CC con mezcla inadecuada (TGA con septum interventricular integro, TAPVR obstruida). 


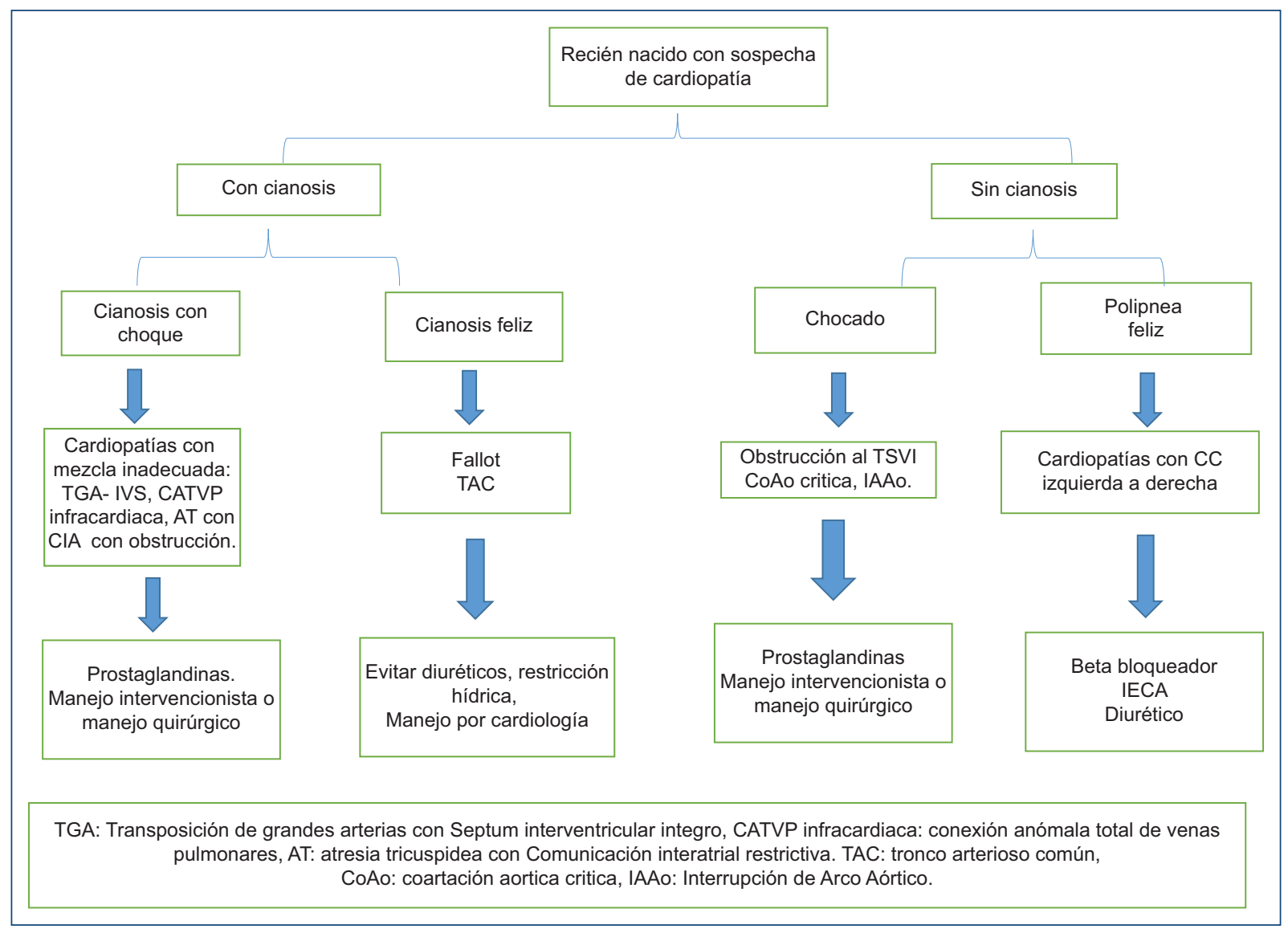

Figura 1. Algoritmo para diagnóstico y manejo del recién nacido con sospecha de cardiopatía.

TGA-IVS: transposición de grandes arterias con septum interventricular íntegro; CATVP infracardiaca: conexión anómala total de venas pulmonares; AT: atresia tricuspídea con comunicación interatrial restrictiva; CIA: comunicación interauricular; TAC: tronco arterioso común; CoAo: coartación aórtica crítica; IAAo: interrupción de arco aórtico; TSVI: tracto de salida del ventrículo izquierdo; CC: cardiopatía congénita; IECA: inhibidores de la enzima convertidora de angiotensina.

Presentan mal estado general, datos de bajo gasto cardiaco y acidosis metabólica; inician de forma brusca en las primeras 72 horas. Los hallazgos en la radiografía de tórax son variados y dependen de la CC; si es una TGA puede ser normal o la típica silueta cardiaca en forma de huevo/ovalada (secundaria al mediastino estrecho) y presentan flujo pulmonar incrementado ${ }^{18}$. Los pacientes con TAPVR obstruida presentan crecimiento atrial y ventricular derecho, el arco de la arteria pulmonar es prominente, la cardiomegalia es mínima o no existe y se perciben datos de hipertensión venocapilar severos ${ }^{19}$. Este tipo de CC se conoce como CCC y para su manejo requieren procedimientos de forma inmediata para retirar la obstrucción ${ }^{16}$. Una mención especial en este grupo es el conducto arterioso, ya que muchas de estas cardiopatías son ductodependientes.
Recordemos que tras el nacimiento el conducto arterioso se cierra de forma funcional entre las $24 \mathrm{y}$ 72 horas y de forma anatómica entre los 10-14 días ${ }^{20}$; para evitar su cierre se utilizan prostaglandinas.

- Cianosis feliz. Cianosis con polipnea sin dificultad respiratoria. La descompensación clínica es más tardía, alrededor de algunas semanas o incluso meses, ya que inicialmente presentan flujo pulmonar y sistémico adecuado, sin embargo, a medida que disminuyen las resistencias vasculares pulmonares presentan datos de sobrecirculación pulmonar, que resulta en insuficiencia $\operatorname{cardiaca}^{16}$ (Fig. 2). Las principales cardiopatías en este grupo son TOF, TAC común y conexión anómala de venas pulmonares no obstruidas. La TOF es la CC cianogena más frecuente y el grado de cianosis depende del grado de obstrucción de la arteria pulmonar, ya sea estenosis o atresia; si es una atresia o 


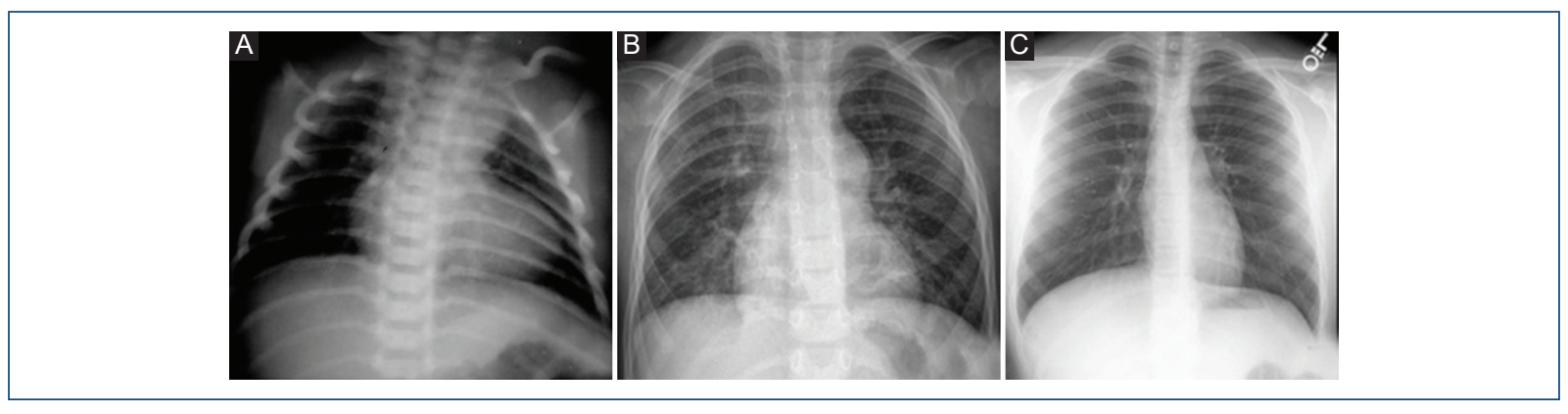

Figura 2. A: radiografía con flujo pulmonar disminuido, típica de un paciente con tetralogía de Fallot. B: radiografía con flujo pulmonar aumentado y tronco de la arteria pulmonar prominente de un paciente con comunicación interventricular. C: radiografía con flujo pulmonar normal.

estenosis severa presentan saturaciones $<75 \%$ sin respuesta al test de hiperoxia y al ser ductodependientes se tratan con prostaglandinas ${ }^{16}$. Si la obstrucción es leve (Fallot rosado) la resistencias vasculares pulmonares disminuirán durante las primeras semanas de vida, provocando mayor diferencia de presión entre ambos ventrículos con sobrecirculación pulmonar y cianosis persistente ${ }^{21}$. En resumen, este grupo de pacientes pueden no tener cianosis al nacer o tener una prueba de oximetría positiva.

- Sin cianosis. Se divide en dos tipos:

- Choque. Presentan importante compromiso cardiovascular, con datos de hipoperfusión y choque cardiogénico. Los síntomas tienen inicio brusco en las primeras 72 horas de vida coincidiendo con el cierre del conducto arterioso ${ }^{22}$; son CCC, ya que el conducto arterioso suple el flujo sistémico desde la arteria pulmonar a la aorta en lesiones con obstrucción importante en el tracto de salida del ventrículo izquierdo como coartación aórtica crítica, HLHS e interrupción de arco aórtico. La sospecha diagnóstica es fundamentalmente clínica, posterior a una exploración física con medición de presión arterial y valoración de pulsos 22 ; ya que en muchas ocasiones los pacientes que presentan diferencial importante entre las presiones arterial preductal y posductal puede sugerir lesión obstructiva del lado izquierdo dependiente de conducto, como una coartación crítica o una estenosis aórtica crítica ${ }^{7}$. La radiografía de tórax muestra cardiomegalia y edema pulmonar. El manejo es integral y requiere diuréticos, corrección de acidosis metabólica ${ }^{22}$, inotrópicos, prostaglandinas y algún procedimiento intervencionista o quirúrgico. El principal diagnóstico diferencial es sepsis, por la similitud con el cuadro clínico.
- Polipnea feliz. Clínicamente se encuentran con polipnea sin otros datos de insuficiencia respiratoria. La presentación clínica es tardía, coincidiendo con la caída de las resistencias pulmonares. La polipnea se acompaña de datos de insuficiencia cardiaca. Por lo general se ocasiona por patologías con cortocircuito de izquierda a derecha, siendo las cardiopatías más frecuentes; como se mencionó anteriormente ocasionan incremento del flujo pulmonar, dilatación de cavidades cardiacas, insuficiencia cardiaca, hipertensión pulmonar inicialmente por hiperflujo $\mathrm{y}$, si no se trata de manera oportuna esta hipertensión, puede progresar a ser secundaria por incremento de las resistencias pulmonares, lo que eventualmente ocasiona síndrome de Einsenmenger ${ }^{23}$. En estos pacientes en forma inicial la administración de oxígeno o vasodilatadores pulmonares incrementa el flujo pulmonar, ocasionando insuficiencia cardiaca y edema agudo pulmonar, por lo que el manejo de este grupo se basa en la disminución de la precarga. La radiografía de tórax muestra cardiomegalia con incremento del flujo pulmonar (Qp > Qs) (Fig. 2). La clasificación con base en la clínica y manejo se enlistan en la figura 1.

\section{¿Cómo se maneja el neonato con cardiopatía congénita?}

Se basa en el grupo al cual pertenece. Si se encuentra en cianosis con choque, son pacientes graves con una CCC y se requiere administración de prostaglandinas. Estas se pueden utilizar por vía intravenosa y en situaciones de urgencias por vía intraósea ${ }^{20,24}$. Si el paciente se encuentra cianótico sin acidosis se deben iniciar de $5-10 \mathrm{ng} / \mathrm{kg} / \mathrm{min}$, si la respuesta es pobre se incrementa la dosis al doble (máximo $100 \mathrm{ng} / \mathrm{kg} / \mathrm{min}$ ) cada 20 minutos hasta tener mejoría clínica con $\mathrm{SaO}_{2}$ 


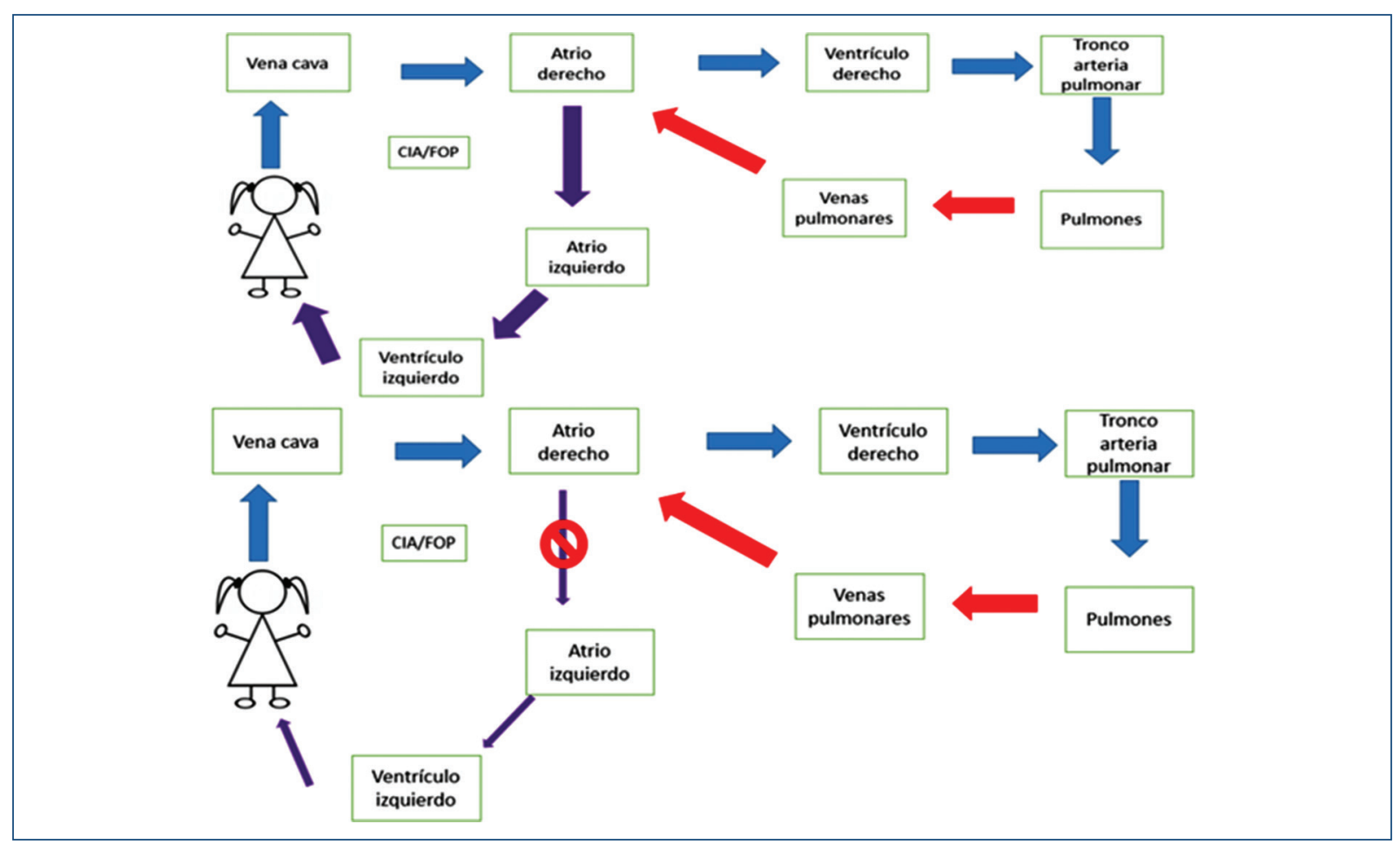

Figura 3. En la parte superior se muestra la fisiopatología de un paciente con Conexión anómala total de venas pulmonares en el cual el gasto sistémico depende del defecto interatrial. En la parte inferior se muestra la fisiopatología de un paciente con CATVP con una CIA pequeña ocasionando bajo gasto cardiaco y edema agudo pulmonar. CATVP infracardiaca: conexión anómala total de venas pulmonares infracardiaca; CIA/FOP: comunicación interauricular /foramen oval permeable.

entre el 75 y el $85 \%$; o saturaciones $<70 \%$ con lactato $<2 \mathrm{mmol} / \mathrm{l}$. En el caso de pacientes cianóticos con pulsos femorales débiles sin acidosis el tratamiento de prostaglandinas es similar. En pacientes con sospecha de CCC y acidosis la dosis inicial de prostaglandinas es de $50 \mathrm{ng} / \mathrm{kg} / \mathrm{min}$, pudiendo requerir dosis elevadas $(100-200 \mathrm{ng} / \mathrm{kg} / \mathrm{min})$ en lo que se decide si requiere manejo intervencionista o quirúrgico. La infusión puede reducirse de $25-50 \mathrm{ng} / \mathrm{kg} / \mathrm{min}$ con mejoría rápida, pero esto usualmente se realiza posterior a la valoración cardiológica ${ }^{20}$. Los parámetros que monitorizar incluyen frecuencia cardiaca, presión arterial, $\mathrm{SaO}_{2}$, frecuencia respiratoria, temperatura y monitoreo gasométrico. Los principales efectos adversos incluyen bradicardia, apneas e hipotensión, por lo que se aconseja mantener a los pacientes en una unidad de cuidados intensivos ${ }^{22}$.

Los pacientes con polipnea feliz tienen CC de hiperflujo pulmonar con riesgo de insuficiencia cardiaca; el manejo se basa en diuréticos de asa, antagonistas de la aldosterona, inhibidores de la enzima convertidora de angiotensina (IECA) y betabloqueadores. Es común que este grupo presente hipertensión pulmonar por hiperflujo, lo cual algunas personas pueden confundir con hipertensión pulmonar por resistencias pulmonares elevadas y administran vasodilatadores pulmonares, provocando mayor hiperflujo, aumento de la precarga, insuficiencia cardiaca y en casos severos edema agudo pulmonar; por ello el manejo se enfoca en controlar la insuficiencia cardiaca.

Los pacientes con cianosis feliz recordemos que son aquellos cuya presentación de la cianosis es tardía (mayor a 5 semanas); no presentan datos de insuficiencia respiratoria y algunos pueden acompañarse de datos de sobrecarga de volumen. Es importante derivarlos a valoración cardiológica para que se indique el manejo adecuado. En la figura 1 se resumen los tipos de CC con base en la presentación clínica y manejo.

\section{Caso clínico 1}

Recién nacido que horas después al nacimiento presenta dificultad respiratoria y datos de bajo gasto. La dificultad respiratoria incrementa requiriendo intubación endotraqueal, presenta $\mathrm{SaO}_{2}<75 \%$ con $\mathrm{FiO}_{2} 100 \%$. No 


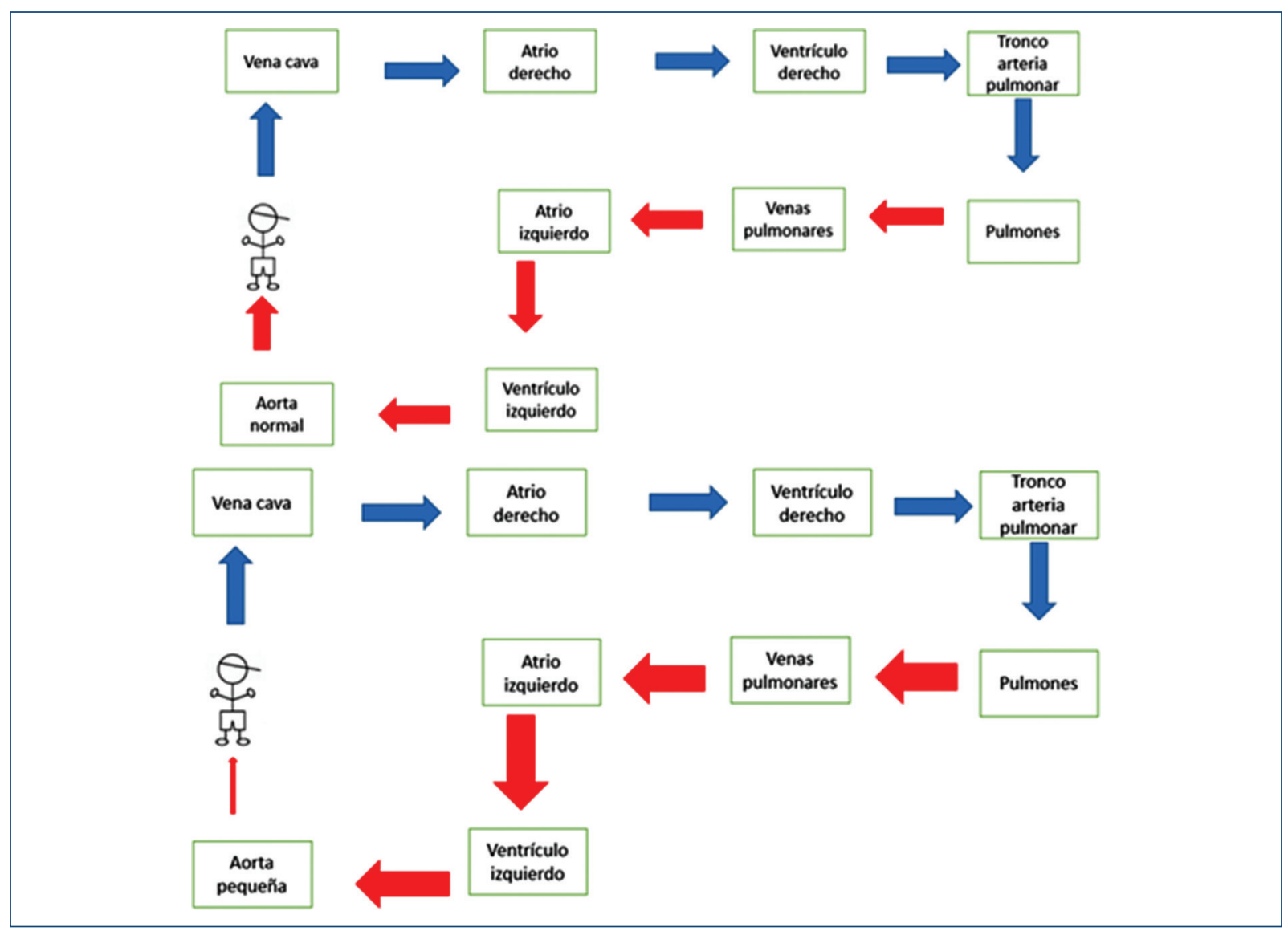

Figura 4. En la parte superior el flujo es normal. La parte inferior muestra una coartación aórtica con disminución del gasto sistémico y por lo tanto con insuficiencia ventricular izquierda y posteriormente edema agudo pulmonar. La radiografía muestra cardiomegalia importante a expensas del ventrículo izquierdo con hiperflujo pulmonar importante.

se auscultan soplos y los pulsos no son palpables. La radiografía sin cardiomegalia y con congestión venosa pulmonar, el ecocardiograma reporta TAPVR con comunicación interatrial restrictiva. Se administran prostaglandinas y se realiza septostomía atrial (Fig. 3).

\section{Caso clínICO II}

Recién nacido de 12 días con datos de bajo gasto, llenado capilar retardado y pulsos no palpables. Radiografía con cardiomegalia e hiperflujo pulmonar; el ecocardiograma reporta coartación aórtica crítica (Fig. 4). Se administran prostaglandinas y se lleva a cabo cateterismo cardiaco urgente.

\section{Etapa lactante}

Las características de las CC se modifican por los cambios hemodinámicos, el más importante es la disminución de las resistencias vasculares pulmonares (posterior a las 3 semanas de edad), lo que provoca un aumento de la sintomatología de las CC con hiperflujo pulmonar.

Las CC se clasifican con base en su fisiopatología. Primero se determina la presencia o no de cianosis y posteriormente el flujo pulmonar en una radiografía de tórax. Para determinar que una radiografía sea adecuada se tiene que valorar si está en inspiración, tráquea y bronquios centrales, se pueden observar algunos discos y cuerpos vertebrales detrás del corazón, lo que indica que la placa tiene una exposición correcta (no es dura ni blanda). Se ve el abdomen superior y los hombros, siendo técnicamente correcto. Los elementos básicos para valorar la silueta cardiaca en una radiografía son localización del hígado y burbuja gástrica, localización del ápex y masa cardiaca, valorar anatomía bronquial, la presencia o no de cardiomegalia y el flujo pulmonar. Este último puede 


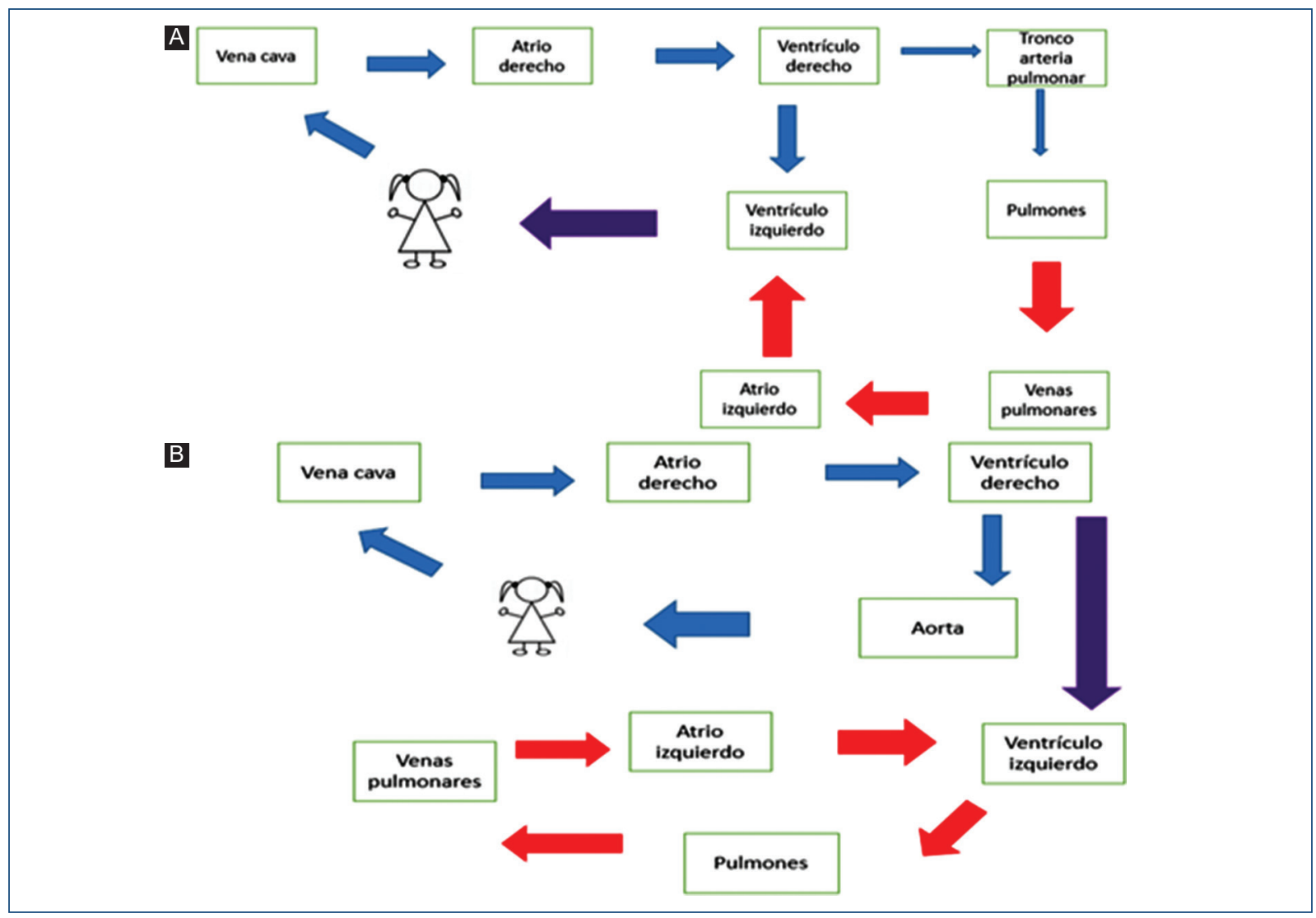

Figura 5. A: fisiopatología de tetralogía de Fallot, se observa disminución en el flujo pulmonar (paso del ventrículo derecho al tronco de la arteria pulmonar) y el cortocircuito de derecha a izquierda a nivel ventricular. B: fisiopatología de transposición de grandes arterias, donde se observa circulación en paralelo con cortocircuito a nivel ventricular.

encontrarse aumentado, disminuido o normal. Un flujo pulmonar incrementado indica un Qp > Qs (Fig. 2), un flujo pulmonar disminuido indica un $Q p<Q s$ (Fig. 2) y un flujo pulmonar normal $Q p=Q$ s, es decir, no se presentan cortocircuitos intracardiacos como en las valvulopatías (Fig. 2).

Ya que conocemos el tipo de flujo pulmonar y la presencia o no de cianosis, las CC se clasifican en:

- Con cianosis, la cual aumenta con el llanto o esfuer-

zo. Se subdividen en dos con base en su flujo pulmonar:

- Aumentado. Son CC con cortocircuito mixto como el TAC común, TGA con comunicación interventricular (Fig. 5). El manejo de estos pacientes por lo general debe estar indicado por el cardiólogo.

- Disminuido. Son CC con cortocircuito de derecha a izquierda con obstrucción de la válvula pulmonar, siendo la principal la TOF (Fig. 5). Deben evitarse los diuréticos y la restricción hídrica.
- Sin cianosis, se dividen en dos subtipos con base en el flujo pulmonar:

- Aumentado. Se encuentran las CC con cortocircuito de izquierda a derecha ( $Q p>Q s)$, tienen flujo pulmonar aumentado y cardiomegalia. La más frecuente es el conducto arterioso, comunicación interventricular (Fig. 6). Estas patologías pueden presentarse con datos clínicos de insuficiencia cardiaca, encontrando en el examen físico datos de dificultad respiratoria con estertores pulmonares. La hepatomegalia es común encontrarla; de hecho, la examinación hepática debe ser obligada en todo paciente en su primer mes de vida con datos de dificultad respiratoria ${ }^{7}$. El manejo incluye betabloqueador, IECA y diurético ${ }^{25}$.

- Flujo pulmonar normal. No tienen cortocircuito ni cardiomegalia, o esta es mínima. Se incluyen valvulopatías como la estenosis aórtica o pulmonar (Fig. 6). 


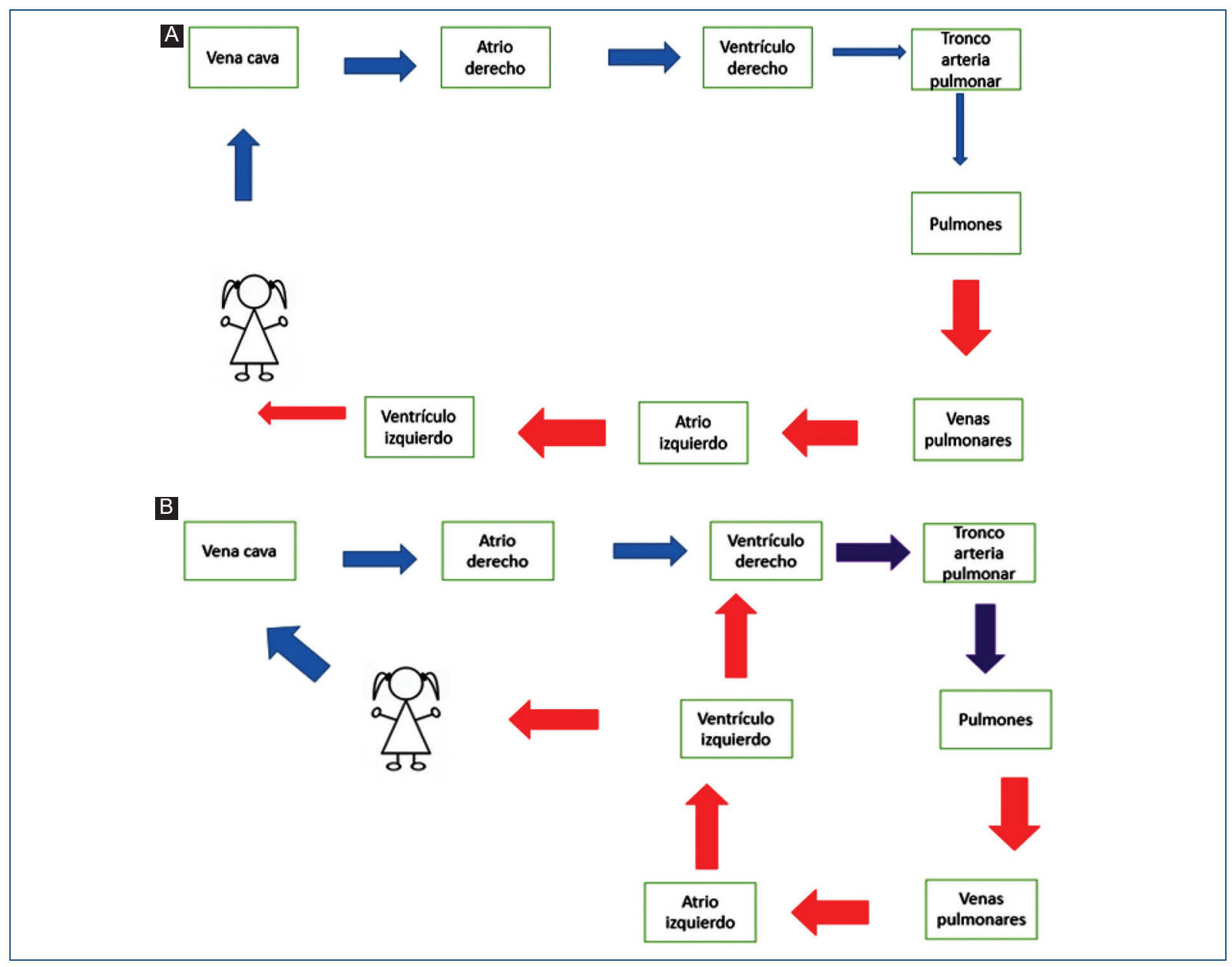

Figura 6. A: fisiopatología de estenosis aórtica, la válvula aórtica está con estenosis, sin embargo al no existir cortocircuito no se afecta el flujo pulmonar. B: comunicación interventricular, se observa cortocircuito de izquierda a derecha que ocasiona incremento del flujo pulmonar $0 p$.

\section{Conclusión}

Las CC constituyen un problema mundial de salud pública ${ }^{2,3}$. A pesar de todos los avances en cardiología pediátrica, lo más importante es que el paciente sea detectado y referido oportunamente. Entre los puntos clave que se pueden concluir se incluyen:

- Ante un paciente con choque cardiogénico en las primeras 72 horas de vida siempre se debe sospechar la existencia de una CCC. Incluso si no se dispone de un estudio ecocardiográfico es esencial iniciar la administración de prostaglandinas ${ }^{25}$.

- Si encontramos flujo pulmonar disminuido en un neonato se requieren prostaglandinas. Si el flujo pulmonar es normal o aumentado acompañado en la exploración física con hepatomegalia y datos de bajo gasto sistémico (choque frío y húmedo) se requiere septostomía atrial. En el caso de que no cuente con bajo gasto sistémico se inicia diurético con IECA.

- Es importante implementar el tamiz cardiológico para la detección oportuna de estos pacientes.

- Los pacientes con CC con cortocircuito de izquierda a derecha por lo general pueden corregirse con una baja mortalidad si se opera en el tiempo adecuado. Sin embargo, un problema común es la derivación tardía de algunos de estos pacientes, lo que hace que se convierta en inoperable $u$ operable con alto riesgo ${ }^{23}$. Por lo tanto, es importante que cada pediatra realice una valoración adecuada con referencia oportuna de estos pacientes a un hospital para su atención.

\section{Financiamiento}

No recibimos ningún tipo de financiamiento. 


\section{Conflicto de intereses}

Los autores declaran no tener conflicto de intereses.

\section{Responsabilidades éticas}

Protección de personas y animales. Los autores declaran que para esta investigación no se han realizado experimentos en seres humanos ni en animales.

Confidencialidad de los datos. Los autores declaran que han seguido los protocolos de su centro de trabajo sobre la publicación de datos de pacientes.

Derecho a la privacidad y consentimiento informado. Los autores han obtenido el consentimiento informado de los pacientes y/o sujetos referidos en el artículo. Este documento obra en poder del autor de correspondencia.

\section{Bibliografía}

1. Van der Bom T, Zomer AC, Zwinderman AH, Meijboom FJ, Bouma BJ, Mulder BJ. The changin epidemiology of congenital heart disease. Nat Rev Cardiol. 2011;8(1):50-60.

2. Calderón-Colmenero J, Cervantes-Salazar JL, Curi-Curi PJ, Ramírez-Marroquín S. Problemática de las cardiopatías congénitas en México. Propuesta de regionalización. Arch Cardiol Mex. 2010;80(2):133-40.

3. Peña-Juárez RA, Medina-Andrade MA. Situación real de las cardiopatías congénitas en dos hospitales públicos del estado de Jalisco. Arch Cardio Mex. 2019;89(4):1-7.

4. Hoffman Jl, Kaplan S. The incidence of congenital heart disease. J Am Coll Cardiol. 2002;39(12):1890-900.

5. Marelli Aj, Mackie AS, Ionescu-Ittu R, Rahme E, Pilote L. Congenital heart disease in the general population: Changing prevalence and age distribution. Circulation. 2007;115(2):163-72.

6. Aranguren Bello HC, Londoño Trujillo D, Troncoso Moreno GA, Dominguez Torres MT, Taborda Restrepo Aa, Fonseca A, et al. Oximetry and neonatal examination for the detection of critical congenital heart disease: a systematic review and meta-analysis. F1000Res. 2019;8:242.

7. Dolbec K, Mick NW. Congenital heart disease. Emerg Med Clin North Am. 2011;29(4):811-27
8. Dawson A, Cassell CH, Riehle-Colarusso T, Scott DG, Tanner JP, Russell SK, et al. Factors associated with late detection of critical congenital heart disease in newborns. Pediatric. 2013;132(3):e604-11.

9. Peña-Juárez RA, Chávez Sáenz JA, García-Canales A, Medina-Andrade M, Martínez-Gonzalez MT, Gutiérrez-Cobian L, et al. Comparación de oxímetros para detección de cardiopatías congénitas críticas. Arch Cardiol Mex. 2019;89(2):172-80.

10. Dhandayuthapani G, Chakrabarti S, Ranasinghe A, Hunt L, Grant D, Martin RP, et al. Short-term outcome of infants presenting of pediatric intensive care unit with new cardiac diagnoses. Congenit Heart Dis. 2010;5:444-49.

11. Nasr VG, DiNardo JA. Pulse oximetry. Pediatr Rev. 2019;40(11):605-8.

12. Teitel $D$. Recognition of undiagnosed neonatal heart disease. Clin Perinatol. 2016;43(1):81-98.

13. Kumar KR, Kirsch RE, Hornik CP. Cardiovascular physiology for intensivists. En: Ungerleider Ross M, editor. Critical heart disease in infants and children. Third edition. Elsevier; 2019. pp 111-133.

14. Marín Rodríguez C, Sánchez Alegre ML, Lancharro Zapata A, Alarcón Rodríguez J. Cuantificación de la circulación pulmonar y sistémica: Qp/ Qs. Qué es, cómo se calcula y para qué sirve. Lo que el radiólogo debe saber. Radiología. 2015;57(5):369-79.

15. Riera Kinkel C. Actualidades en el tratamiento quirúrgico de las cardiopatías congenitas. Rev Mex Pediatr. 2010;77(5):214-23.

16. Desai K, Rabinowitz EJ, Epstein S. Physiologic diagnosis of congenital heart disease in cyanotic neonates. Curr Opin Pediatr. 2019;31:274-83.

17. Martín Escribano P. Semiología de la cianosis. Arch de bronconeumol 1974; 10(2): 80-81)

18. Sarris GE, Balmer C, Bonou P, Comas JV, da Cruz E, Di Chiara L, et al. Clinical guidelines for the management of patients with transposition of the great arteries with intact ventricular septum. Eur J Cardiothorac Surg. 2017:51(1):e1-e32.

19. Patiño Bahena EJ, Calderón Colmenero J, Buendía Hernández A, Attie F. Conexión anómala total de venas pulmonares. En: Attie M, editor. Cardiología pediátrica. 2. a edición. Panamericana; 2012. pp. 92- 101.

20. Singh $Y$, Mikrou $P$. Use of prostaglandins in duct-dependent congenital heart conditions. Arch Dis Child Educ Pract Ed. 2018;103(3):137-40.

21. Aortopulmonary septal defects and patent ductus arteriosus, total anomalous pulmonary venous return, transposition of the great arteries, tetralogy of Fallot with and without pulmonary atresia, hypoplastic left heart syndrome. En: Nichols DG, Ungerleider RM, Spevak PJ, et al. Critical heart disease in infants and children. 2nd ed. Philadelphia, PA: Mosby; 2006. Págs. 652-60.

22. Maroto C, Camino M, Girona JM, Malo P, Romera G, Zunzunegui JL. Recién nacido con sospecha de cardiopatía congénita. Protocolos Diagnóstico Terapéuticos de la AEP: Neonatología. Rev Esp Cardiol. 2008:54:49-66.

23. Vijayalakshmi IB. Evaluation of left to right shunts by pediatrician: How to follow, when to refer for intervention? Indian J Pediatr. 2015;82(11):1027-32.

24. Mikrou $P$, Singh $A$, Singh $Y$. Prostaglandin infusion Guideline. Eur J Pediatr 2015; 154:700-7.

25. Parvathi UI, Moreno Guillermo E, Caneo LF, Faiz T, Shekerdeian LS, lyer KS. Management of late presentation congenital heart disease. Cardiol Young. 2017;27:6:s31-39. 